\section{(O) OPEN ACCESS}

\title{
Validity of a three-variable Juvenile Arthritis Disease Activity Score in children with new-onset juvenile idiopathic arthritis
}

\author{
Flora Mcerlane, ${ }^{1,2}$ Michael W Beresford, ${ }^{2,3}$ Eileen M Baildam, ${ }^{3}$ S E Alice Chieng, ${ }^{4}$ \\ Joyce E Davidson, ${ }^{5}$ Helen E Foster, ${ }^{6}$ Janet Gardner-Medwin ${ }^{5}$ Mark Lunt, ${ }^{1}$ \\ Lucy R Wedderburn, ${ }^{7}$ Wendy Thomson, ${ }^{1}$ Kimme L Hyrich, ${ }^{1}$ on behalf of the Childhood \\ Arthritis Prospective Study (CAPS)
}

\begin{abstract}
Handling editor Tore K Kvien
- Additional material is published online only. To view please visit the journal online (http://dx.doi.org/10.1136/ annrheumdis-2012-202031).
\end{abstract}

For numbered affiliations see end of article

\section{Correspondence to}

Kimme Hyich, Arthritis Research UK Epidemiology Unit, Centre for Musculoskeletal Research, Institute of Inflammation and Repair, The University of Manchester, Manchester Academic Health Science Centre, Room 2.800 Stopford Building, Oxford Road, Manchester, M13 9PT, UK; Kimme.hyrich@ manchester.ac.uk

Received 16 May 2012 Revised 14 November 2012 Accepted 27 November 2012 Published Online First 20 December 2012

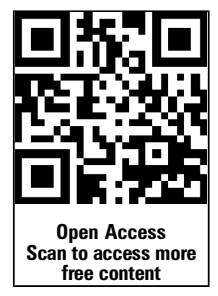

To cite: Mcerlane $F$, Beresford MW, Baildam EM, et al. Ann Rheum Dis 2013;72:1983-1988.

\section{ABSTRACT}

Objectives To investigate the validity and feasibility of the Juvenile Arthritis Disease Activity Score (JADAS) in the routine clinical setting for all juvenile idiopathic arthritis (JIA) disease categories and explore whether exclusion of the erythrocyte sedimentation rate (ESR) from JADAS (the 'JADAS3') influences correlation with single markers of disease activity.

Methods JADAS-71, JADAS-27 and JADAS-10 were determined at baseline for an inception cohort of children with JIA in the Childhood Arthritis Prospective Study. JADAS3-71, JADAS3-27 and JADAS3-10 were determined using an identical formula but with exclusion of ESR. Correlation of JADAS with JADAS3 and single measures of disease activity/severity were determined by category.

Results Of 956 eligible children, sufficient data were available to calculate JADAS-71, JADAS-27 and JADAS-10 at baseline in 352 (37\%) and JADAS3 in 551 (58\%). The median (IQR) JADAS-71, JADAS-27 and JADAS-10 for all 352 children was 11 (5.9-18), 10.4 (5.7-17) and 11 (5.9-17.3), respectively. Median JADAS and JADAS3 varied significantly with the category (Kruskal-Wallis $p=0.0001$ ), with the highest values in children with polyarticular disease patterns. Correlation of JADAS and JADAS3 across all categories was excellent. Correlation of JADAS71 with single markers of disease activity/severity was good to moderate, with some variation across the categories. With the exception of ESR, correlation of JADAS3-71 was similar to correlation of JADAS-71 with the same indices.

Conclusions This study is the first to apply JADAS to all categories of JIA in a routine clinical setting in the UK, adding further information about the feasibility and construct validity of JADAS. For the majority of categories, clinical applicability would be improved by exclusion of the ESR.

\section{INTRODUCTION}

Inflammatory arthritis, one of the most common chronic illnesses of childhood, occurs in approximately $10: 100000$ children each year, ${ }^{1}$ with the majority subsequently diagnosed with juvenile idiopathic arthritis (JIA). JIA is an umbrella term, summarising the internationally recognised International League Against Rheumatism (ILAR) classification system for the markedly heterogeneous chronic paediatric arthritides. ${ }^{2}$
The term 'disease activity' refers to the overall burden of inflammatory disease at a particular point in time. Accurate assessment of disease activity is central to improving clinical outcomes in JIA, since the prevention of joint damage and associated long-term disability requires prompt recognition and control of inflammatory disease. The heterogeneous nature of JIA ensures that no single measure can reliably capture overall disease activity in all patients. A feasible, valid and reproducible assessment of disease activity is integral to the design of new clinical studies and clinical trials, improving the efficiency and effectiveness of clinical research and enabling between-study comparisons.

The Juvenile Arthritis Disease Activity Score (JADAS) is a new composite disease activity score specific to JIA. ${ }^{4}$ It is simple to calculate using four variables measured in the clinical setting: active joint count (AJC), physician global assessment (physician global), parent global evaluation (parent global) and erythrocyte sedimentation rate (ESR). JADAS has been shown to be feasible, with good construct validity, discriminant validity and responsiveness to clinically important change. ${ }^{4}$ However, many of the original validation data were derived from clinical trials, with a predominance of polyarticular-course disease and very few children with enthesitis-related or psoriatic JIA. An observational study of JADAS was published in 2012, but, despite a relatively large sample size, the validity and feasibility of JADAS was difficult to ascertain in all ILAR categories because of the relative infrequency of some of the categories in the population. ${ }^{5}$ Furthermore, it is recognised that the ESR is not routinely measured in all clinical settings, largely because of the stress and difficulty associated with regular blood testing. The ESR is more likely to be measured in children on medication requiring blood monitoring, meaning that children with persistent oligoarticular JIA (the most common category) are less likely to have an ESR available than children with systemic onset JIA or a polyarticular disease pattern. Therefore the JADAS as proposed, which includes the ESR, is less likely to be a measure with clinical feasibility and applicability.

The objectives of this analysis were to (1) investigate the validity and clinical feasibility of JADAS as 
a summary of disease activity at the time of first presentation using data collected in the routine clinical setting in all ILAR categories of JIA and (2) explore whether exclusion of the ESR from JADAS (the 'JADAS3') influences correlation with single markers of disease activity.

\section{METHODS}

\section{Study population}

Children in this analysis were participants in the Childhood Arthritis Prospective Study (CAPS), an ongoing prospective inception cohort study launched in 2001. ${ }^{6}$ CAPS aims to provide long-term outcome data on children with new-onset inflammatory arthritis receiving routine specialist care in the UK. In brief, children aged $<16$ years with a new diagnosis of inflammatory arthritis present for at least 2 weeks, presenting to one of five UK paediatric rheumatology centres (Liverpool, Manchester, Newcastle, Glasgow and Great Ormond Street), are invited to participate. Exclusion criteria are septic arthritis and arthritis related to malignancy, trauma or connective tissue disease (systemic lupus erythematosus, juvenile dermatomyositis or mixed connective tissue disease). The study was approved by the UK Northwest Multicentre Research Ethics Committee. Written informed consent has been obtained from the parent(s)/ guardians of all participating children, and all able children have provided written assent.

\section{Data collection}

Data for this analysis have been collated from the CAPS database and include information from medical records and interview with the child/family. The AJC, limited joint count (LJC) and $10 \mathrm{~cm}$ physician global visual analogue scale (VAS) are documented by the paediatric rheumatologist at the first (baseline) paediatric rheumatology visit (baseline study visit). The rheumatologist is asked to assign a predicted ILAR category if the suspected/confirmed diagnosis is JIA, where possible. For children presenting within the first 6 months of the disease, this was considered a 'predicted' category only. The parent/child is asked to complete a Childhood Health Assessment Questionnaire (CHAQ) validated for use in the UK population, ${ }^{8}$ including a $10 \mathrm{~cm}$ parent global VAS and $10 \mathrm{~cm}$ pain VAS. A paediatric rheumatology research nurse interviews the parent(s) and child within 3 months of the initial visit and extracts demographic and clinical data from the medical records. Similar data are collected at 6 months and then annually to 5 years, including a confirmation of the underlying diagnosis and ILAR subtype.

\section{Analysis}

All children with a confirmed physician's diagnosis of JIA were included in this analysis. For children whose baseline study visit was within the first 6 weeks of disease onset, only those with further study follow-up confirming a diagnosis of JIA were included. JADAS-71, JADAS-27 and JADAS-10 were determined for all children at the time of first presentation using the formula published by Consolaro et al. ${ }^{4}$

The JADAS is the linear sum of the scores of its four components (range $=0-101$ for JADAS-71, 0-57 for JADAS-27, and 040 for JADAS-10):

$$
\begin{aligned}
\mathrm{JADAS}= & \mathrm{AJC}(71,27 \text { or } 10 \text { joints })+\text { physician global }(10 \mathrm{cmVAS}) \\
& + \text { parent global }(10 \mathrm{cmVAS})+\mathrm{ESR}
\end{aligned}
$$

JADAS-71 includes a complete joint count (71 joints), whereas JADAS-27 and JADAS-10 include reduced joint counts
(JADAS-27: 27 joints including cervical spine, elbows, wrists, 1st-3rd metacarpophalangeals, proximal interphalangeals, hips, knees and ankles; JADAS-10: any involved joint to a maximum of 10). There is no weighting of joints. To avoid excessive weight in the overall index, the ESR is converted into a $0-10$ scale as follows: (ESR $(\mathrm{mm} / \mathrm{h})-20) / 10$. ESR values $<20$ are allocated a score of 0 , and ESR results $>120$ score 10 on the scale. As the ESR may not have been measured on the same day as the rheumatology examination, we allowed a window of up to 30 days before or after the first visit.

Calculation of the JADAS3 was identical with that for the full JADAS with the exclusion of ESR (range=0-91 for JADAS3-71, 0-47 for JADAS3-27, and 0-30 for JADAS3-10). To study the construct validity of JADAS, the correlation of JADAS-71, JADAS-27 and JADAS-10 with one another and with single measures of disease activity and severity (AJC, LJC, physician global, parent global, ESR, pain score and CHAQ) were determined for the entire cohort and within each ILAR category at baseline. Although the ILAR category can change with time, this study used the ILAR category allocated at baseline to correlate with the timing of recording the components of the JADAS. All correlations were calculated using Spearman's rank statistic. Correlations were considered high, moderate or low at $>0.7$, 0.4-0.7 and <0.4, respectively. A similar analysis was carried out for JADAS3, limited at first to children with the fourvariable JADAS available and then repeated in the larger cohort with sufficient data to calculate JADAS3. All analyses were performed using Stata V.10.0.

\section{RESULTS}

\section{Baseline characteristics and availability of JADAS}

In January 2012, a total of 956 children with a confirmed diagnosis of JIA and ILAR category available at baseline had been recruited to CAPS. The median age at disease onset was 6.6 years (IQR 2.6-10.6), median disease duration at diagnosis was 5.5 months (IQR 2.9-11.8), and 65\% were female (table 1).

It was not possible to calculate JADAS at the time of first presentation for all children (table 1), with complete data in all four components being available in just 352/956 children at baseline (37\%). All children had an AJC documented, but 17\% were missing the physician global, 32\% missing the parent global, and 36\% missing the ESR (table 2). The ESR had the widest range of missing data across all ILAR categories (recorded in $82 \%$ of children with systemic onset, 59\% with oligoarticular and 44\% with enthesitis-related JIA). Exclusion of the ESR from JADAS allowed calculation of JADAS3 in 551/ 956 children $(58 \%)$.

Children with sufficient data available to calculate JADAS-71, JADAS-27 and JADAS-10 had significantly higher LJC and physician global scores (Kruskal-Wallis $\mathrm{p}<0.05$ ) than those without, and there was a trend towards higher pain and CHAQ scores in this subgroup (table 1). Children with sufficient data to calculate JADAS3-71, JADAS3-27 and JADAS3-10 had significantly higher physician global scores than the remainder of the cohort. There was a trend towards a higher proportion of the cohort with JADAS available having systemic onset JIA, but this did not reach statistical significance $\left(\chi^{2} \mathrm{p}=0.12\right)$. There was no significant difference in ILAR subtype distribution in the cohorts with and without JADAS3 available $\left(\chi^{2} \mathrm{p}=0.2\right)$.

\section{JADAS and JADAS3 results by ILAR subtype of JIA}

The correlation of JADAS and JADAS 3 across all ILAR categories was excellent (table 3). 
Table 1 Baseline characteristics of children with and without available items for the Juvenile Arthritis Disease Activity Score (JADAS)

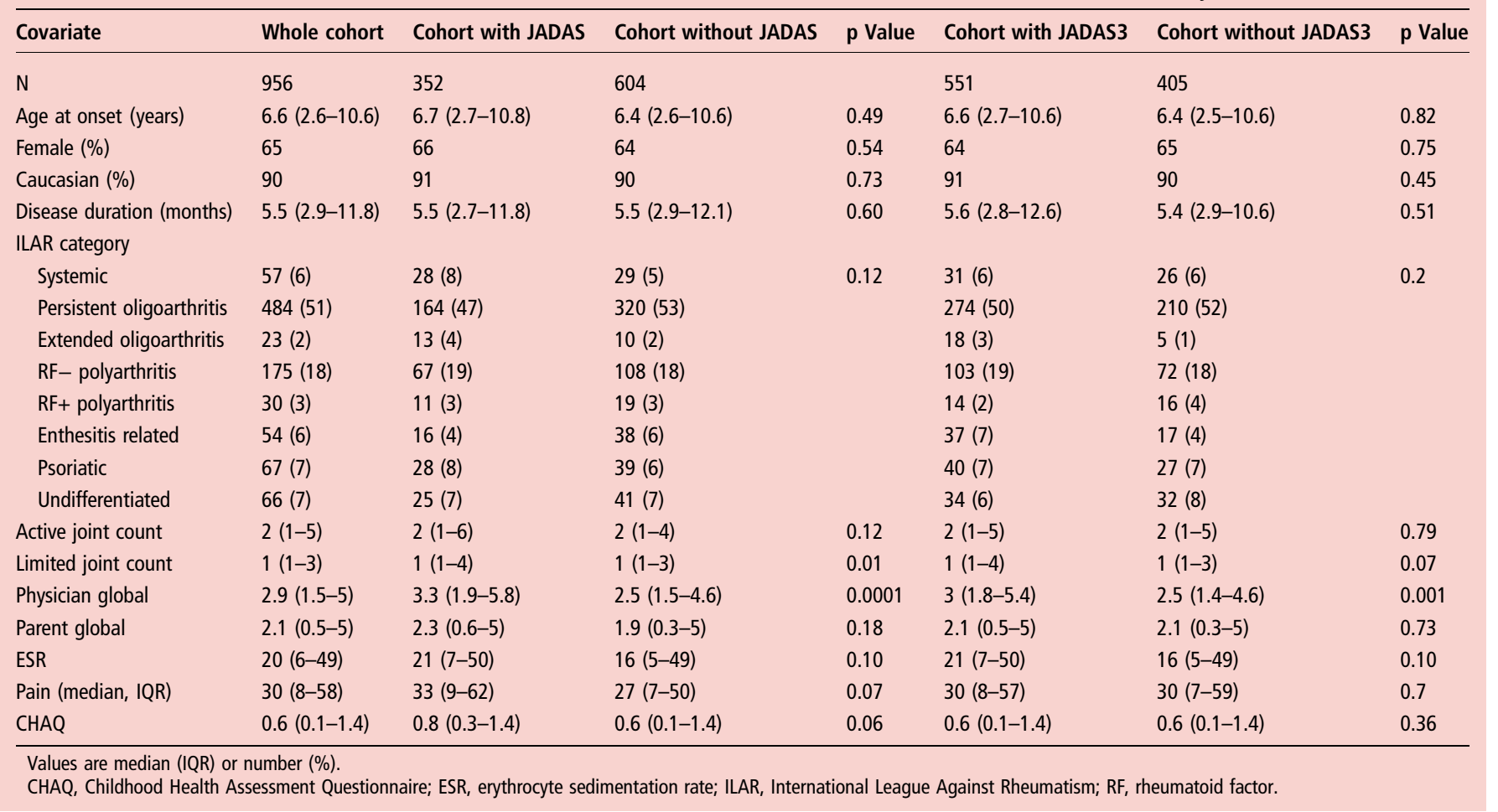

The median (IQR) JADAS-71, JADAS-27 and JADAS-10 for all 352 children was 11 (IQR 5.9-18), 10.4 (5.7-17) and 11 (5.917.3), respectively (table 4). Correlation between the three JADAS results was high, ranging from $0.87-1.0$ across the categories (see online supplementary table S1). As expected, median JADAS3-71, JADAS3-27 and JADAS3-10 results were lower than median JADAS-71, JADAS-27 and JADAS-10, with the greatest difference in the systemic onset category (table 4). The JADAS and JADAS3 results were minimally different in the extended oligoarticular and rheumatoid factor $(\mathrm{RF})+$ polyarticular categories.

Median JADAS and JADAS3 results varied significantly with the ILAR category (Kruskal-Wallis $\mathrm{p}=0.0001$ ) (table 4 and see online supplementary table S2).

\section{Construct validity of JADAS and JADAS3 in routine clinical practice}

Correlations of JADAS-71 and JADAS3-71 with individual surrogates for disease activity are presented in table 5. Correlation of JADAS-71 with AJC was high, with the exception of persistent oligoarticular JIA $(r=0.47)$. Correlation with the remaining three variables included within the score varied across the categories. On average, correlation with the ESR was the lowest, ranging from low to moderate across the categories. Correlation of JADAS-71 with other markers of disease activity/ severity, including LJC, CHAQ and pain, ranged from low to high/moderate across the categories. Low numbers rendered correlation coefficients difficult to interpret in the extended oligoarticular and RF+ polyarticular categories.

With the exception of the ESR, correlation of JADAS3-71 with individual measures of disease activity was very similar to correlation of JADAS-71 with the same indices. Correlation of JADAS3-71 with the ESR was low in all categories with the exception of undifferentiated JIA.

As the cohort of children with JADAS available was slightly weighted towards those with more severe disease, we also undertook further validation of JADAS3 in all children with available data $(n=551)$ (see online supplementary table S2). Compared with the cohort with the full JADAS available, the

Table 2 Proportion of recorded values within individual components of JADAS at first visit to paediatric rheumatology

\begin{tabular}{llllllllll}
\hline Component & $\begin{array}{l}\text { Whole } \\
\text { cohort }\end{array}$ & Systemic & $\begin{array}{l}\text { Persistent } \\
\text { oligoarthritis }\end{array}$ & $\begin{array}{l}\text { Extended } \\
\text { oligoarthritis }\end{array}$ & $\begin{array}{l}\text { RF (-) } \\
\text { polyarthritis }\end{array}$ & $\begin{array}{l}\text { RF (+) } \\
\text { polyarthritis }\end{array}$ & $\begin{array}{l}\text { Enthesitis } \\
\text { related }\end{array}$ & $\begin{array}{l}\text { Psoriatic } \\
\text { arthritis }\end{array}$ & \multicolumn{1}{c}{ Undifferentiated } \\
\hline $\mathrm{N}$ & 959 & 57 & 484 & 23 & 175 & 30 & 54 & 67 & 66 \\
$\begin{array}{l}\text { Active joint } \\
\text { count }\end{array}$ & $959(100)$ & $57(100)$ & $484(100)$ & $23(100)$ & $175(100)$ & $30(100)$ & $54(100)$ & $67(100)$ & $66(100)$ \\
$\begin{array}{l}\text { Physician } \\
\text { global }\end{array}$ & $794(83)$ & $42(74)$ & $412(85)$ & $23(100)$ & $142(81)$ & $21(70)$ & $48(89)$ & $57(85)$ & $49(74)$ \\
$\begin{array}{l}\text { Parent global } \\
\text { ESR }\end{array}$ & $647(68)$ & $37(65)$ & $315(65)$ & $18(78)$ & $124(71)$ & $19(63)$ & $41(76)$ & $47(70)$ & $46(70)$ \\
\hline
\end{tabular}

Values are number (\%).

ESR, erythrocyte sedimentation rate; JADAS, Juvenile Arthritis Disease Activity Score. 
Table 3 Correlation of JADAS and JADAS3 at first presentation to paediatric rheumatology

\begin{tabular}{|c|c|c|c|c|}
\hline & $\begin{array}{l}\text { Number } \\
\text { of } \\
\text { patients }\end{array}$ & $\begin{array}{l}\text { JADAS-71 } \\
\text { and } \\
\text { JADAS3-71 }\end{array}$ & $\begin{array}{l}\text { JADAS-27 } \\
\text { and } \\
\text { JADAS3-27 }\end{array}$ & $\begin{array}{l}\text { JADAS-10 } \\
\text { and } \\
\text { JADAS3-10 }\end{array}$ \\
\hline Whole cohort & 352 & 0.96 & 0.96 & 0.96 \\
\hline Systemic onset & 28 & 0.93 & 0.94 & 0.92 \\
\hline $\begin{array}{l}\text { Persistent } \\
\text { oligoarthritis }\end{array}$ & 164 & 0.96 & 0.96 & 0.96 \\
\hline $\begin{array}{l}\text { Extended } \\
\text { oligoarthritis }\end{array}$ & 13 & 0.94 & 0.91 & 0.95 \\
\hline RF- polyarthritis & 67 & 0.93 & 0.93 & 0.89 \\
\hline $\mathrm{RF}+$ polyarthritis & 11 & 0.98 & 0.96 & 0.96 \\
\hline $\begin{array}{l}\text { Enthesitis-related } \\
\text { arthritis }\end{array}$ & 16 & 0.94 & 0.91 & 0.92 \\
\hline Psoriatic arthritis & 28 & 0.95 & 0.95 & 0.94 \\
\hline $\begin{array}{l}\text { Undifferentiated } \\
\text { arthritis }\end{array}$ & 25 & 0.99 & 0.99 & 0.98 \\
\hline
\end{tabular}

JADAS, Juvenile Arthritis Disease Activity Score; RF, rheumatoid factor.

JADAS3 results in the larger cohort were lower overall, but showed a similar level of correlation across the study sample.

\section{DISCUSSION}

This paper describes JADAS across all ILAR categories of JIA within a large 'real-world' dataset reflecting routine clinical care and is the first to place a benchmark of disease activity at initial presentation to paediatric rheumatology in all ILAR categories. The correlation between JADAS-71, JADAS-27 and JADAS-10 was excellent for all categories. Median JADAS-71 ranged from 7.5 to 20 and was significantly higher among children with polyarticular pattern disease or systemic onset JIA. The correlation of JADAS-71 with individual markers of disease activity was moderate to good for the whole cohort, with some variation between categories. The clinical applicability of JADAS-71, JADAS-27 and JADAS-10 was limited by availability of data.

We were unable to calculate a four-variable JADAS in the majority of children in our cohort because of missing data. Unlike a clinical trial, where a protocol would be strictly followed to ensure that all data points are collected at each time point, this observational study collected 'real-world' data as recorded in the medical case notes. For many children, missing data resulted from blood samples not being taken for ESR measurement. To explore this, we tested the validity of excluding the ESR from JADAS (labelled JADAS3-71, JADAS3-27 and JADAS3-10). This increased the proportion of patients for whom a score could be calculated from 37\% to 58\%. Further validation of the JADAS3 in the cohort limited to those with JADAS available, as well as in the larger cohort, resulting in a more representative sample of our overall study cohort, found similar results across the ILAR categories.

A large proportion of children had the physician global and parent global assessments missing. Children with sufficient data available to calculate JADAS or JADAS3 had significantly higher physician global scores than those without. This implies that physicians may be more likely to collect all data points in children with more severe disease. One possible explanation is that data may be collected more often if there is a perceived clinical consequence-for example, access to biological medications. The recently published Arthritis and Musculoskeletal Alliance (ARMA)/British Society of Paediatric and Adolescent

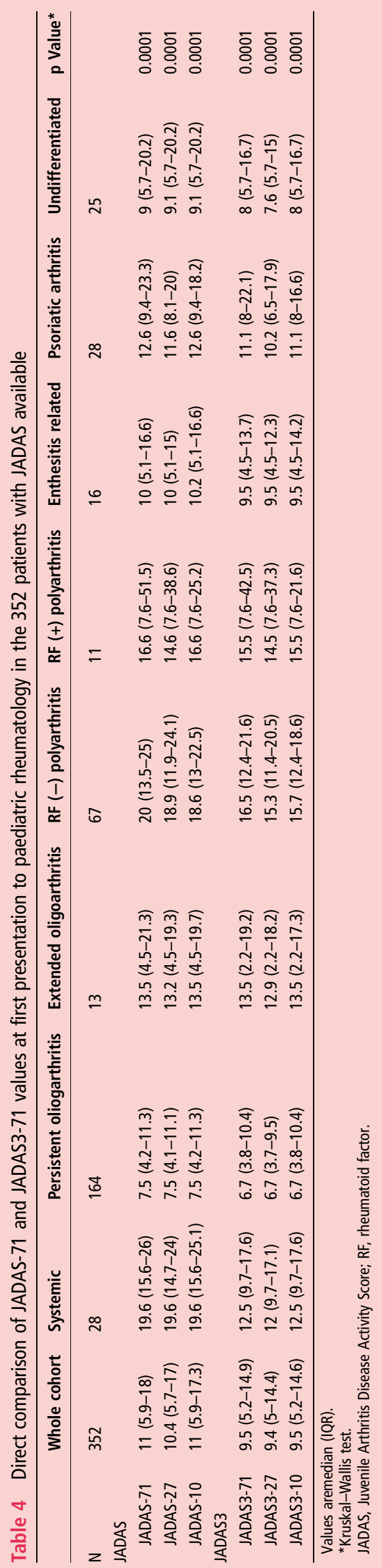


Table 5 Correlation of JADAS-71 and JADAS3-71 with individual measures of disease activity in the 352 patients with JADAS available

\begin{tabular}{|c|c|c|c|c|c|c|c|c|c|}
\hline & $\begin{array}{l}\text { Whole } \\
\text { cohort }\end{array}$ & Systemic & $\begin{array}{l}\text { Persistent } \\
\text { oliogarthritis }\end{array}$ & $\begin{array}{l}\text { Extended } \\
\text { oligoarthritis }\end{array}$ & $\begin{array}{l}\mathrm{RF}(-) \\
\text { polyarthritis }\end{array}$ & $\begin{array}{l}\mathrm{RF}(+) \\
\text { polyarthritis }\end{array}$ & $\begin{array}{l}\text { Enthesitis } \\
\text { related }\end{array}$ & $\begin{array}{l}\text { Psoriatic } \\
\text { arthritis }\end{array}$ & Undifferentiated \\
\hline $\mathrm{N}$ & 352 & 28 & 164 & 13 & 67 & 11 & 16 & 28 & 25 \\
\hline \multicolumn{10}{|l|}{ JADAS-71 } \\
\hline AJC & 0.75 & 0.79 & 0.47 & 0.8 & 0.73 & 0.95 & 0.78 & 0.82 & 0.85 \\
\hline Physician global & 0.73 & 0.53 & 0.73 & 0.55 & 0.7 & 0.66 & 0.67 & 0.5 & 0.83 \\
\hline Parent global & 0.57 & 0.62 & 0.71 & 0.75 & 0.33 & 0.39 & 0.68 & 0.39 & 0.65 \\
\hline ESR & 0.53 & 0.6 & 0.33 & 0.01 & 0.48 & 0.4 & 0.43 & 0.41 & 0.69 \\
\hline LC & 0.51 & 0.71 & 0.34 & 0.49 & 0.29 & 0.76 & 0.67 & 0.61 & 0.47 \\
\hline Pain & 0.48 & 0.37 & 0.58 & 0.57 & 0.34 & 0.04 & 0.81 & 0.47 & 0.54 \\
\hline CHAQ & 0.58 & 0.46 & 0.57 & 0.71 & 0.44 & 0.54 & 0.71 & 0.6 & 0.63 \\
\hline \multicolumn{10}{|l|}{ JADAS3-71 } \\
\hline AJC & 0.79 & 0.86 & 0.53 & 0.88 & 0.83 & 0.95 & 0.82 & 0.79 & 0.87 \\
\hline Physician global & 0.72 & 0.41 & 0.73 & 0.54 & 0.64 & 0.7 & 0.61 & 0.59 & 0.85 \\
\hline Parent global & 0.60 & 0.72 & 0.75 & 0.68 & 0.34 & 0.38 & 0.81 & 0.4 & 0.64 \\
\hline ESR & 0.33 & 0.36 & 0.1 & -0.27 & 0.22 & 0.29 & 0.16 & 0.17 & 0.62 \\
\hline LJC & 0.53 & 0.79 & 0.38 & 0.47 & 0.33 & 0.77 & 0.57 & 0.58 & 0.42 \\
\hline Pain & 0.51 & 0.46 & 0.62 & 0.54 & 0.31 & 0.1 & 0.8 & 0.48 & 0.5 \\
\hline CHAQ & 0.58 & 0.5 & 0.59 & 0.65 & 0.47 & 0.53 & 0.74 & 0.5 & 0.63 \\
\hline
\end{tabular}

AJC, active joint count; CHAQ, Childhood Health Assessment Questionnaire; ESR, erythrocyte sedimentation rate; JADAS, Juvenile Arthritis Disease Activity Score; LC, limited joint count; RF, rheumatoid factor.

Rheumatology 'Standards of Care for Children and Young People with JIA' stipulate that all children and young people with JIA should have core outcome variables (including therefore the components of JADAS) measured at each review. ${ }^{10}$ Collection of these data as part of routine care is challenging, but essential to ensure important targets of clinical remission are achieved on and off medication. ${ }^{11}$

JADAS-71, JADAS-27 and JADAS-10 correlated highly across all categories. Reduced joint count tools have not yet been validated in all ILAR categories, so this in itself is an interesting finding. ${ }^{12}$ Since recording of active disease in every joint can be technically difficult and time-consuming, reduced joint counts may potentially be useful in routine practice. The DAS28, a 28 -joint reduced count, is an effective assessment of disease activity in rheumatoid arthritis, ${ }^{13}{ }^{14}$ although its use in adult psoriatic arthritis has been criticised, with particular reference to exclusion of the ankle and foot. ${ }^{15}$ Reduced joint counts have been shown to correlate with whole joint counts in clinical trials of patients with JIA, but have not been validated in the routine clinical setting in all subtypes. ${ }^{12}$ Routine application of the 27 -joint reduced joint count would improve the ease and speed of examination, but has the potential to miss clinically relevant information.

Correlation of JADAS-71 with individual markers of disease activity was moderate to good for the whole cohort. These findings are similar to the results of previous validation studies. $^{4} 516$ This study demonstrated significant variation across the ILAR categories. For example, in children with oligoarticular JIA, the moderate correlation with AJC reflected a limited range of active joints associated with a wide range of global scores. Variation between the categories may reflect the heterogeneity of the disease (particularly if extra-articular features (enthesitis or rash) dominate the physician and parental global scores) and may relate, in part, to the low numbers within some categories.

Correlation of JADAS-71 with the ESR was moderate overall (0.53), varying markedly between categories. Correlation was moderate in children with systemic onset JIA (0.6), and moderate to low in all other categories, except undifferentiated JIA, a highly diverse and difficult to interpret category of children.

Correlation of JADAS3-71 with the ESR was low in all ILAR categories. This may relate in part to the fact that we allowed a window of 30 days to collect the ESR at baseline, reflecting current clinical practice. In the UK, blood samples are often not checked at the first visit, but are taken when the child returns for treatment, such as a joint injection under general anaesthetic or intravenous therapy. Therefore, it is possible that the result may have been influenced on some occasions by treatment. The infrequent use of oral steroids limits the likelihood of this scenario.

Biomarkers are readily available, non-subjective assays, potentially adding useful information to other more subjective but more responsive disease activity surrogates. ${ }^{17}$ However, the analysis of a number of rheumatoid arthritis composite indices has suggested that acute phase reactants provide little information on actual disease activity on top of that provided by the combination of several clinical indices. ${ }^{18}$ Furthermore, it is likely that biomarkers are less responsive to disease flares in oligoarticular disease than in polyarticular disease. Exclusion of the ESR (JADAS3) did not reduce the correlation of JADAS with other surrogates for disease activity. JADAS3 may therefore be a pragmatic alternative to JADAS in the absence of an ESR measurement in the clinical setting.

Baseline data were used in this study, as disease activity is often the most severe at first presentation. The use of baseline data allowed this analysis to benchmark the severity of disease at this time point, using baseline JADAS. However, even at this point, the ESR was not measured in all children, reflecting routine clinical practice in the UK. This resulted in a limited number of children for whom JADAS could be calculated, particularly in some ILAR categories. The small population of children with JADAS available represents a possible limitation of this study, with respect to applying our results across the wider JIA population.

A further limitation of our analysis may be the use of ILAR category as assigned at the first presentation. It has previously 
been noted that the ILAR category can change over time, ${ }^{9}$ particularly in children with oligoarticular pattern disease. Since this was within the first 6 months of disease for the majority of children, the assigned ILAR category can only be considered a 'predicted' ILAR category, and further validation of the JADAS and JADAS3 result at later time points is also required. The reliability and sensitivity to change of JADAS3 could not be ascertained in this cohort of children at first presentation.

\section{CONCLUSION}

In conclusion, this study is the first to apply JADAS to all ILAR categories of JIA in the routine clinical setting at the time of first presentation, adding further information about the feasibility and construct validity of JADAS in the clinical setting. For the majority of categories, clinical feasibility would be improved by exclusion of the ESR, allowing a composite measure of disease activity at the bedside. An amended score (JADAS3), which omits the ESR, correlates well with JADAS.

Routine collection of the core outcome variables would permit calculation of JADAS3 at the bedside, offering the clinical team an opportunity to improve the quality of clinical care through a more precise, less subjective summary of disease activity, which can be more accurately compared with previous measurements and clearly communicated to children and their families.

\section{Author affiliations \\ ${ }^{1}$ Arthritis Research UK Epidemiology Unit, University of Manchester, Manchester, UK ${ }^{2}$ Department of Translational Medicine, Institute of Child Health, University of Liverpool, Liverpool, UK \\ ${ }^{3}$ Department of Paediatric Rheumatology, Alder Hey Children's Hospital NHS Foundation Trust, Liverpool, UK \\ ${ }^{4}$ Department of Rheumatology, Royal Manchester Children's Hospital, Manchester, UK \\ ${ }^{5}$ Department of Paediatric Rheumatology, Royal Hospital for Sick Children, University of Glasgow, Glasgow, UK \\ ${ }^{6}$ Musculoskeletal Research Group, Institute Cellular Medicine, Newcastle University and Paediatric Rheumatology, Great North Children's Hospital, Newcastle upon Tyne, UK \\ ${ }^{7}$ Rheumatology Unit, Institute of Child Health, UCL and Great Ormond Street Hospital NHS Trust, UCL Institute of Child Health, London, UK}

Acknowledgements The authors would like to thank the CAPS contributors for collecting the data (Dr Eileen Baildam, Mrs Carol Lydon, Ms Lynsey Brown, Mrs Olivia Lloyd, Dr Joyce Davidson, Dr Janet Gardner-Medwin, Mrs Kay Riding, Ms Vicki Price, Mrs Jane Sim, Mrs Maureen Todd, Mrs Elizabeth Friel, Ms Jadranka Zelenovic, Mrs Sharon Watson, Ms Vikki Gould, Professor Lucy Wedderburn, Mrs Julie Jones, Mrs Alexandra Meijer, Ms Natasha Makengo, Dr Helen Foster, Dr Mark Friswell, Mr Michael Eltringham, Mrs Lucy Cook, Mrs Susan Tremble, Mrs Debbie Wade, Mrs Liang Qiao, Dr Alice Chieng, Ms Joanne Buckley, Mrs Ann McGovern, Mrs Annette Duggan, Professor Tauny Southwood), Peter Ward for co-ordinating the study, Mark Lay for running the database, and Professor Alan Silman for his help in establishing the study. FM is funded by a Barbara Ansell Fellowship from Arthritis Research UK.

Contributors $\mathrm{FM}, \mathrm{KLH}$ and WT were responsible for data collection, data cleaning and data analysis. MWB, EMB, SEAC, JED, HEF, JG-M, ML and LRW contributed to the final analysis.

Funding Barbara Ansell Fellowship, Arthritis Research UK.

Competing interests None.

Ethics approval UK Northwest Multicentre Research Ethics Committee.
Patient consent Obtained.

Provenance and peer review Not commissioned; externally peer reviewed.

Open Access This is an Open Access article distributed in accordance with the Creative Commons Attribution Non Commercial (CC BY-NC 3.0) license, which permits others to distribute, remix, adapt, build upon this work non-commercially, and license their derivative works on different terms, provided the original work is properly cited and the use is non-commercial. See: http://creativecommons.org/ licenses/by-nc/3.0/

\section{REFERENCES}

1 Symmons DP, Jones M, Osborne J, et al. Pediatric rheumatology in the UK: data from the British Pediatric Rheumatology Group National Diagnostic Register. J Rheumatol 1996:23:1975-80.

2 Petty RE, Southwood TR, Baum J, et al. Revision of the proposed classification criteria for juvenile idiopathic arthritis: Durban, 1997. J Rheumatol 1998;25:1991-4.

3 Petty RE, Southwood TR, Manners P, et al. International League of Associations for Rheumatology classification of juvenile idiopathic arthritis: second revision, Edmonton, 2001. J Rheumatol 2004;31:390-2.

4 Consolaro A, Ruperto N, Bazso A, et al. Development and validation of a composite disease activity score for juvenile idiopathic arthritis. Arthritis Rheum 2009:61:658-66

5 Nordal EB, Zak M, Aalto K, et al. Validity and predictive ability of the juvenile arthritis disease activity score based on CRP versus ESR in a Nordic population-based setting. Ann Rheum Dis 2012;71:1122-7.

6 Adib N, Hyrich K, Thornton J, et al. Association between duration of symptoms and severity of disease at first presentation to paediatric rheumatology: results from the Childhood Arthritis Prospective Study. Rheumatology(Oxford) 2008:47:991-5.

7 Hyrich KL, Lal SD, Foster HE, et al. Disease activity and disability in children with juvenile idiopathic arthritis one year following presentation to paediatric rheumatology. Results from the Childhood Arthritis Prospective Study. Rheumatology (Oxford) 2010;49:116-22

8 Nugent J, Ruperto N, Grainger J, et al. The British version of the Childhood Health Assessment Questionnaire (CHAQ) and the Child Health Questionnaire (CHQ). Clin Exp Rheumatol 2001;19(4 Suppl 23):S163-7.

9 Nordal E, Zak M, Aalto K, et al. Ongoing disease activity and changing categories in a long-term nordic cohort study of juvenile idiopathic arthritis. Arthritis Rheum 2011;63:2809-18.

10 ARMA/BSPAR Standards of Care for Children and Young People with JIA. http:/l arma.uk.net/local-groups/care-documents/juvenile-idiopathic-arthritis/2010 (cited 21 Mar 2012).

11 Beresford MW, Cleary AG, Foster HE, et al. Comment on: developing standards of care for patients with juvenile idiopathic arthritis. Rheumatology (Oxford) 2010:49:2227-9.

12 Bazso A, Consolaro A, Ruperto N, et al. Development and testing of reduced joint counts in juvenile idiopathic arthritis. J Rheumatol 2009;36:183-90.

13 Fuchs HA, Brooks RH, Callahan LF, et al. A simplified twenty-eight-joint quantitative articular index in rheumatoid arthritis. Arthritis Rheum 1989:32:531-7.

14 Fuchs HA, Pincus T. Reduced joint counts in controlled clinical trials in rheumatoid arthritis. Arthritis Rheum 1994:37:470-5.

15 Leeb BF, Andel I, Sautner J, et al. The Disease Activity Score in 28 joints in rheumatoid arthritis and psoriatic arthritis patients. Arthritis Rheum 2007:57:256-60.

16 Ringold S, Bittner R, Neogi T, et al. Performance of rheumatoid arthritis disease activity measures and juvenile arthritis disease activity scores in polyarticular-course juvenile idiopathic arthritis: analysis of their ability to classify the American College of Rheumatology pediatric measures of response and the preliminary criteria for flare and inactive disease. Arthritis Care Res 2010;62:1095-102.

17 Magni-Manzoni S, Cugno C, Pistorio A, et al. Responsiveness of clinical measures to flare of disease activity in juvenile idiopathic arthritis. Clin Exp Rheumatol 2005;23:421-5.

18 Aletaha D, Nell VP, Stamm T, et al. Acute phase reactants add little to composite disease activity indices for rheumatoid arthritis: validation of a clinical activity score. Arthritis Res Ther 2005;7:R796-806. 